\title{
Qualité des eaux de surface dans la vallée du Sourou : cas des rivières Mouhoun, Sourou, Debe et Gana au Burkina Faso
}

\author{
Dayéri DIANOU ${ }^{1 *}$, Boubacar SAVADOGO ${ }^{1}$, Dramane ZONGO ${ }^{1}$, \\ Tidiane ZOUGOURI ${ }^{2}$, Jean Noel PODA ${ }^{1}$, Hortense BADO ${ }^{3}$ et Francis ROSILLON ${ }^{4}$ \\ ${ }^{I}$ Institut de Recherche en Sciences de la Santé, Centre National de la Recherche Scientifique et \\ Technologique, 03 BP 7192 Ouagadougou, Burkina Faso. \\ ${ }^{2}$ Laboratoire National d'Analyse des Eaux, Ministère de l'Environnement et du Développement Durable, \\ Ouagadougou, Burkina Faso. \\ ${ }^{3}$ Convention pour la promotion d'un développement durable (COPROD), Ouagadougou, Burkina Faso. \\ ${ }^{4}$ Département des Sciences et Gestion de l'Environnement, Université de Liège, Campus d'Arlon, Belgique. \\ *Auteur correspondant, E-mail : dayerid@yahoo.fr;Tél : +22650335684/503643 37 ; \\ GSM : +22676239961.
}

\section{RESUME}

Dans le cadre d'une gestion participative des eaux dans la vallée du Sourou, un suivi de la qualité des eaux des rivières Mouhoun, Sourou, Débé et Gana a été réalisé en Novembre 2007, Février 2008 et Juin 2008. Les analyses physico-chimiques et bactériologiques ont mis en évidence la relation entre les activités anthropiques et la qualité physico-chimique et bactériologique des plans d'eau particulièrement sur certains sites. Les concentrations de nitrates relativement plus élevées dans les eaux à Di aval $(9,2 \mathrm{mg} / \mathrm{L})$ et Débé aval (5,6 mg/L) en début d'hivernage sont liées principalement aux pratiques agricoles aux abords des plans d'eau. L'abreuvement intensif d'animaux au point de contrôle du Gana et les activités domestiques à Toma-île sont la cause de fortes contaminations fécales des eaux : $12200 \mathrm{UFC} / 100 \mathrm{ml}$ pour Escherichia coli et $12800 \mathrm{UFC} / 100$ $\mathrm{ml}$ pour les eaux du Gana contre 1260 et 5000 UFC/100 ml respectivement pour les eaux en aval de Toma-île. Les pollutions apparaissent plus prononcées en période sèche et d'harmattan et particulièrement en début d'hivernage. D'une manière générale, les eaux des petits réservoirs comme celui du Gana sont plus sensibles aux pollutions que celles des grands réservoirs (Mouhoun, Sourou, Débé) caractérisés par une qualité différenciée des eaux d'un emplacement à un autre.

(C) 2011 International Formulae Group. All rights reserved.

Mots clés : Eau, Pollution, Bactériologie, Physico-chimie, Rivière, Sourou.

\section{INTRODUCTION}

L'eau constitue un atout essentiel pour le développement de tout pays. Comme le souligne Klaus Toepfler, directeur général du Programme des Nations Unies pour l'Environnement (PNUE), cité par Kouam et al. (2006), "L'eau est étroitement liée à la santé, l'agriculture, l'énergie et la biodiversité. Sans progrès dans le domaine de l'eau, il sera difficile voir impossible d'atteindre les autres Objectifs du Millénaire pour le Développement". Face aux pressions combinées de l'augmentation accélérée de la demande et de la détérioration continue de la 
qualité de l'eau en particulier dans les pays en développement (PED), une gestion efficace et efficiente des ressources en eau doit prendre en compte non seulement la rareté de la ressource eau mais également sa qualité. En clair, une maîtrise de l'eau dans ses différents compartiments est le support essentiel du développement socioéconomique d'un pays en améliorant la santé de ses populations (Phillips et al., 2006; Julien, 2006 ; Wellens et al., 2008a; Wellens et al., 2008b). De nombreux pays développés notamment européens l'ont compris à travers la mise en œuvre de structures telles que la Directive Cadre sur l'Eau (DCE) adoptée en 2000 par le parlement Européen et dont l'objectif principal est de prévenir la détérioration des masses d'eau à travers un suivi de la qualité de ces eaux. En France, l'Office de l'eau et des milieux aquatiques (Onema) créé en 2006 est chargé des études et recherches de portée générale notamment le suivi et la surveillance de la qualité de l'eau et des milieux aquatiques et constitue un enjeu majeur dans le contexte de l'atteinte du bon état ou du bon potentiel prôné par la DCE (Richard et Rieu, 2009). D'autres structures comme les contrats de rivière ont été créées en France et en Belgique. Celles-ci fonctionnent depuis plus de 20 ans et s'inscrivent dorénavant dans la politique européenne de l'eau. Les pays en développement en général et le Burkina Faso en particulier, ne disposent malheureusement pas de données fiables et constituées de leurs ressources en eau (MEA, 2001 ; Somé et al., 2008; MAHRH, 2011). Les données sont éparses et sont généralement acquises lors de la mise en œuvre de grands projets (MEA, 2001). De même, il n'existe pas des normes nationales bien définies et complètes pour les ressources en eau. On se réfère en général, avec tout ce que cela comporte comme écart, aux normes de l'Organisation Mondiale de la Santé (OMS) et aux normes de l'Union
Européenne (MEA, 2001; Kouam et al., 2006 ; Somé et al., 2008).

Au Burkina Faso, la rivière Sourou est un affluent du principal fleuve du pays, le Mouhoun (Volta Noire). Les eaux de cette rivière alimentent la principale zone hydroagricole du pays (vallée du Sourou). La permanence de l'eau a renforcé ses multiples usages qui sont la culture irriguée, la pêche, l'élevage, le maintien de la biodiversité, l'alimentation en eau de boisson des populations insulaires, les activités domestiques et récréatives (Traoré, 2003; Poda et al., 2006 ; Poda, 2007). L'Autorité de Mise en Valeur du Sourou (AMVS), les coopératives agricoles, les villages îles sur les berges de la rivière, les hippopotames protégés, les cultures commerciales de contresaison, les populations d'oiseau... donnent une importance particulière à cette zone logée dans un milieu soudanien où l'eau est souvent rare.

Les fortes pressions sur les masses d'eau pour différents usages ont entrâné leur dégradation continue au plan quantitatif et qualitatif. Cette situation a conduit à la mise en place d'un cadre pour une gestion intégrée et participation des eaux dans la vallée, le "Contrat de Rivière Sourou".

La présente étude se situe dans ce cadre et a pour objectifs d'évaluer la qualité physico-chimique et bactériologique des eaux de surface dans la vallée en fonction des périodes de l'année et d'en établir les liens avec les usages et pratiques autour des plans d'eau. A cet effet, nous avons caractérisé les eaux des principaux cours d'eau dans la vallée pendant la période sèche, au début et à la fin de la saison pluvieuse en 2007-2008.

\section{MATERIEL ET METHODES}

\section{Site d'étude}

La Vallée du Sourou est située dans le Nord-Ouest du Burkina Faso (Latitude Nord : 
$12^{\circ} 00^{\prime} ; 13^{\circ} 30^{\prime}$ et Longitude Ouest : $3^{\circ} 00^{\prime}$; $4^{\circ} 30^{\prime}$ ) (Figure 1).

La Rivière Sourou est un cours d'eau orienté du nord vers le sud et long d'environ 55 kilomètres entre l'extrême Oué et Léri (site de sa confluence avec le fleuve Mouhoun: Volta Noire), (Figure 2). Elle constitue le principal cours d'eau dans la vallée du Sourou. Depuis la construction d'infrastructures à la confluence de Léri en 1984, la rivière Sourou draine d'importantes quantités d'eau $\left(600000000 \mathrm{~m}^{3}\right)$ à travers la vallée d'environ 615000 ha avec pour résultante, la présence de villages insulaires sur le cours d'eau. Le Sourou se comporte comme un affluent (basses eaux) / défluent (hautes eaux) du fleuve Mouhoun. En période de crue du Mouhoun, une partie des eaux remonte le lit du Sourou qui coule alors du sud au nord. Par contre en période d'étiage, les eaux retournent au Mouhoun, le Sourou coule alors du nord vers le sud. Il s'en suit un décalage entre la période des hautes eaux dans le Mouhoun et le Sourou. La côte maximale des eaux est en général atteinte en novembredécembre, c'est-à-dire un à deux mois après la fin de la saison pluvieuse (Traoré, 2003; Bethemont et al., 2003). Ce jeu complexe du flux/reflux des eaux dans le Sourou est rendu possible à cause de sa déclivité longitudinale faible de l'ordre de 0,5\%o (Leprun, 1968).

La permanence et l'étendue des masses d'eau font de la vallée du Sourou une excellente zone agricole aménagée par l'Etat pour répondre à la demande nationale et régionale en ressources alimentaires notamment de riz. Ainsi, entre les villages traditionnels, se sont successivement mises en place de grandes coopératives: Guiédougou en 1967, puis Niassan et Débé en 1990. La demande de plus en plus forte en eau et les multiples usages ont sans conteste des répercussions importantes sur la qualité des eaux de surface qui sont d'une importance vitale pour le développement de la vallée.

\section{Prélèvements et analyses des eaux}

Le réseau de mesure de la qualité des eaux de surface dans la vallée du Sourou est constitué de 10 points de contrôle (sites) répartis le long des cours d'eau (Figure 2). Le choix de ces sites tient compte des multiples usages des eaux de surface aux différents endroits de la vallée (Tableau 1).

Trois campagnes de prélèvement ont été effectuées en 2007 et 2008 à différentes périodes de l'année: (1) fin de saison pluvieuse (Novembre 2007), (2) période fraîche/chaude et d'harmattan (Février 2008) et (3) début de saison pluvieuse (Juin 2008).

En plus des analyses physicochimiques effectuées sur le terrain, un échantillonnage est réalisé en triple dans des flacons stériles pour les analyses bactériologiques suivies des autres analyses physico-chimiques au laboratoire.

Les eaux de surface des différents points de contrôle sont échantillonnées le même jour dans la partie médiane du cours d'eau au cours de chaque campagne.

Les paramètres physico-chimiques généraux ( $\mathrm{pH}$, Matières en suspension, Conductivité électrique, Oxygène dissous et Turbidité) ont été mesurés in situ à l'aide de sondes électrochimiques spécifiques. Les nitrates ont été évalués sur le site à l'aide de bandelettes réactives puis au laboratoire par colorimétrie. Les autres paramètres chimiques (DCO, Nitrites, Ammonium et Phosphates) ont été déterminés par le Laboratoire National d'Analyse des Eaux (LNAE) selon des méthodes standardisées adaptées de Rodier (1996).

Les paramètres bactériologiques (Escherichia coli et Coliformes fécaux) ont été évalués sur le milieu chromogène Rapid'Ecoli par la méthode d'étalement sur boîte de Pétri à $44,5{ }^{\circ} \mathrm{C}$ pendant $24-48$ heures dans une étuve thermostatée au laboratoire. 


\section{Analyses statistiques}

Les données obtenues ont été soumises à une analyse de variance et les différences entre moyennes observées entre périodes et entre sites ont été comparées suivant le test de Newman-Keuls au seuil de 5\% avec le logiciel XLSTAT 7.5.2. Les corrélations au seuil de $5 \%$ entre les différentes variables ont été analysées en relation avec les périodes d'échantillonnage et les sites. Les paramètres mesurés ont été évalués suivant les normes du Système d'Evaluation de la Qualité de l'Eau des cours d'eau (SEQ - Eau, France 2003) (Tableau 2). Les normes OMS (WHO, 2004) pour la qualité des eaux de boisson ont également été prises en compte du fait que les eaux de surface dans la vallée, outre les autres usages, alimentent également certaines populations en eau de boisson, notamment celles des villages situés aux abords ou sur le cours d'eau Sourou (Toma-île, Yaran, Wèrè, Touroukoro, ...) (Traoré, 2003 ; Dianou et al., 2004 ; Koukounari et al., 2007).

\section{RESULTATS}

\section{Paramètres physico-chimiques}

Les Tableaux 3 et 4 présentent l'analyse de variance et les paramètres physico-chimiques des échantillons d'eau pour les trois campagnes. Dans l'ensemble, les milieux sont bien oxygénés et présentent des concentrations faibles au niveau des paramètres physico-chimiques analysés au regard des normes définies pour la qualité des eaux des cours d'eau (Tableau 2) (SQE-Eau, 2003). En dehors de l'ammonium et de la conductivité, ces paramètres apparaissent respectivement en lien avec la période d'échantillonnage $(p<0,05)$ et le site $(p<0,01)$ (Tableau 3). Toutefois, l'effet conjoint de la période et du site influence significativement les paramètres mesurés ( $\mathrm{p}<0,01$; Tableau 3 ). La période sèche et de forte pression sur les eaux (Février : période 2) et celle de début de saison pluvieuse (Juin : période 3) présentent les valeurs les plus élevées respectivement pour la conductivité $(\mathrm{p}<0,01)$, la DCO ( $\mathrm{p}<$ $0,05)$, les nitrates $(\mathrm{p}<0,01)$, les nitrites $(\mathrm{p}<$ $0,01)$ et les ortho-phosphates $(\mathrm{p}<0,01)$ pour tous les sites confondus. Des valeurs prononcées sont observées en période sèche sur le site du Gana, respectivement pour la conductivité (353 $\mu \mathrm{S} / \mathrm{cm})$, la DCO (603 mg $\left.\mathrm{O}_{2} / \mathrm{L}\right)$ et les ortho-phosphates $\left(0,98 \mathrm{mg} \mathrm{PO}_{4}{ }^{3-}\right.$ /L) (Tableau 4). En outre, ce site présente les $\mathrm{pH}$ les plus bas pour les périodes $1(6,2)$ et 2 $(5,5)$. Les turbidités observées en début d'hivernage sont significativement plus élevées que celles des autres périodes pour la presque totalité des points de contrôle excepté le Gana ( $p<0,05$; Tableau 4). Les eaux de Di aval et Toma-île aval révèlent les plus fortes concentrations de nitrates en début de saison d'hivernage avec respectivement 9,2 et $5,6 \mathrm{mg} / \mathrm{L}$ (Tableau 4). Ces eaux comportent également des teneurs relativement importantes pour cet élément en période sèche (4,2 et 4,6 mg/L) à l'image de celles de Débé aval $(4,7 \mathrm{mg} / \mathrm{L})$. En outre, une corrélation positive entre les teneurs en nitrates et la turbidité des eaux est observée à Di aval ( $\mathrm{r}=$ $0,94 ; \mathrm{p}<0,05)$. Quant aux matières en suspension, elles apparaissent plus prononcées en fin de saison pluvieuse (Novembre: période 1) pour l'ensemble des points de contrôle $(\mathrm{p}<0,05)$ avec les valeurs les plus prononcées enregistrées sur le site du Gana (896 mg/L) (Tableau 4). Sur un plan d'ensemble, pour tous les sites et les périodes confondus, les analyses ont mis en évidence une corrélation positive entre la turbidité et les matières en suspension $(\mathrm{r}=0,999 ; \mathrm{p}<0,05)$ et une corrélation négative $(\mathrm{r}=-0,88 ; \mathrm{p}<$ $0,05)$ entre la turbidité et l'oxygène dissous.

\section{Paramètres bactériologiques}

Les résultats (Tableaux 5 et 6) indiquent une contamination fécale plus ou moins prononcée des eaux aux différents points de contrôle au regard des normes de 
qualité pour les eaux de baignade (Tableau 2) (SQE-Eau, 2003).

De fortes concentrations sont observées particulièrement au point de contrôle du Gana pendant la période sèche pour Escherichia coli (12200 UFC/100 ml) et les coliformes fécaux (12800 UFC/100 ml) et également en fin de saison d'hivernage (4445 UFC/100 ml pour Escherichia coli et $10856 \mathrm{UFC} / 100 \mathrm{ml}$ pour les coliformes fécaux). Le même facies est observé à Toma-île aval en début de saison d'hivernage (1260 UFC/100 $\mathrm{ml}$ pour Escherichia coli et $5000 \mathrm{UFC} / 100 \mathrm{ml}$ pour les coliformes fécaux) et en saison sèche (393 $\mathrm{UFC} / 100 \mathrm{ml}$ et $1000 \mathrm{UFC} / 100 \mathrm{ml}$ respectivement pour ces deux paramètres) (Tableau 6). L'analyse de variance indique toutefois que les résultats observés ne sont pas liés à la période d'échantillonnage ( $\mathrm{p}<0,05$; Tableau 5). Par contre, le point de contrôle (site) influence grandement les résultats observés ( $\mathrm{p}<0,01$; Tableau 5).

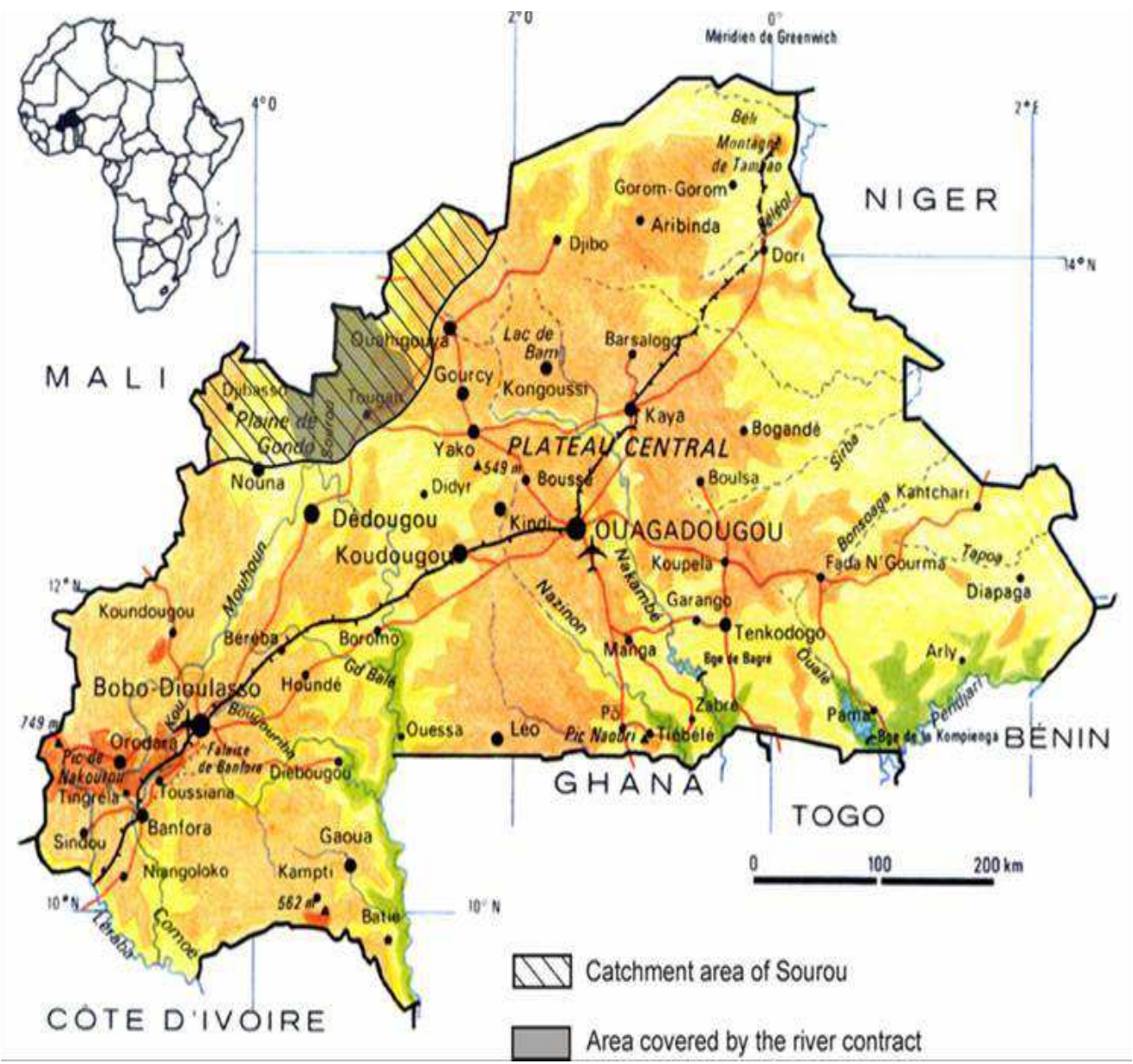

Figure 1 : Zone couverte par le Contrat de Rivière au Sourou, Burkina Faso (Source : Rosillon et Bado, 2006).

Zone couverte par le Contrat de Rivière.

WV Zone du Sourou 


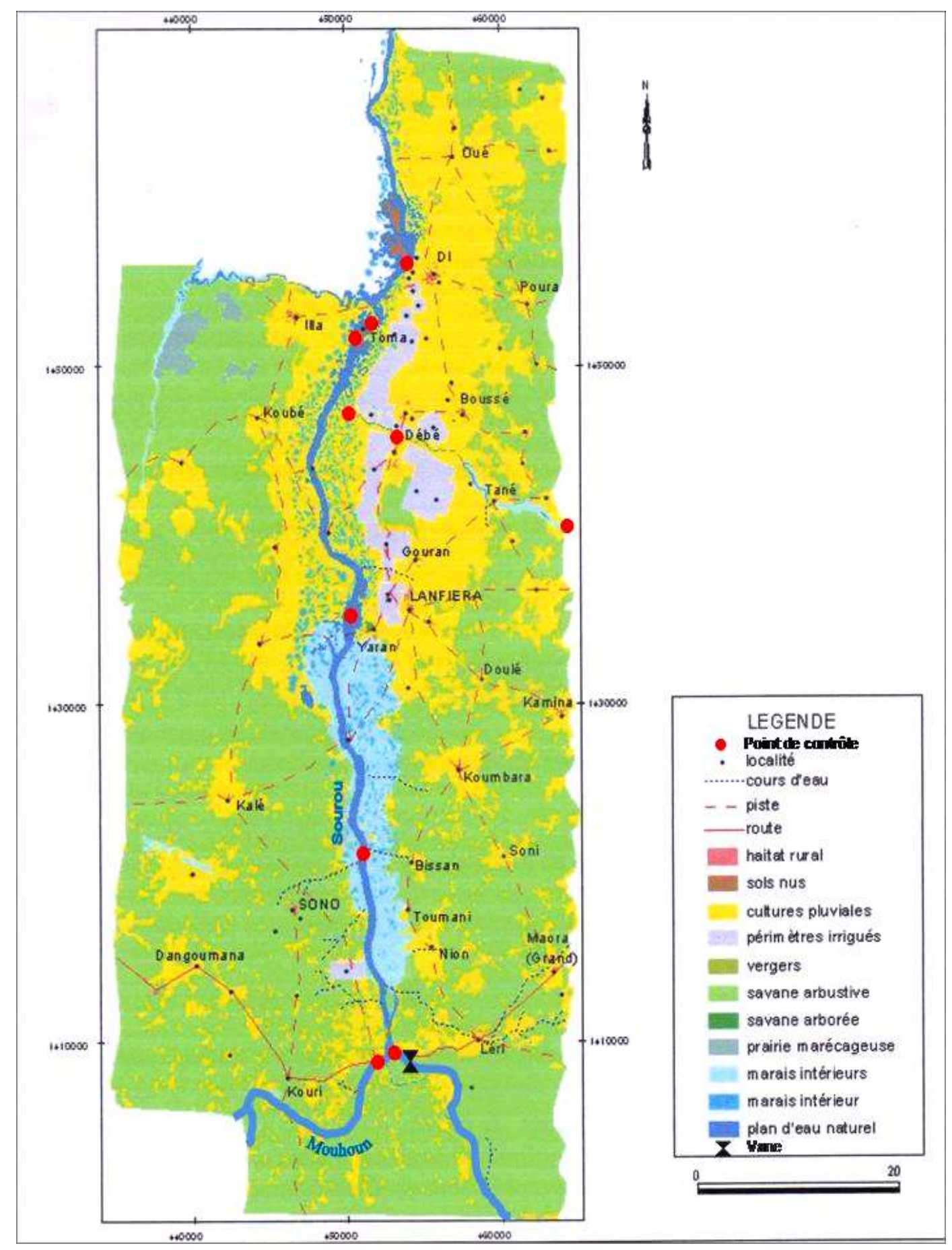

Figure 2: Sites de contrôle de la qualité des eaux de surface dans la vallée du Sourou (Source fond de carte: Rosillon et Bado, 2006). 


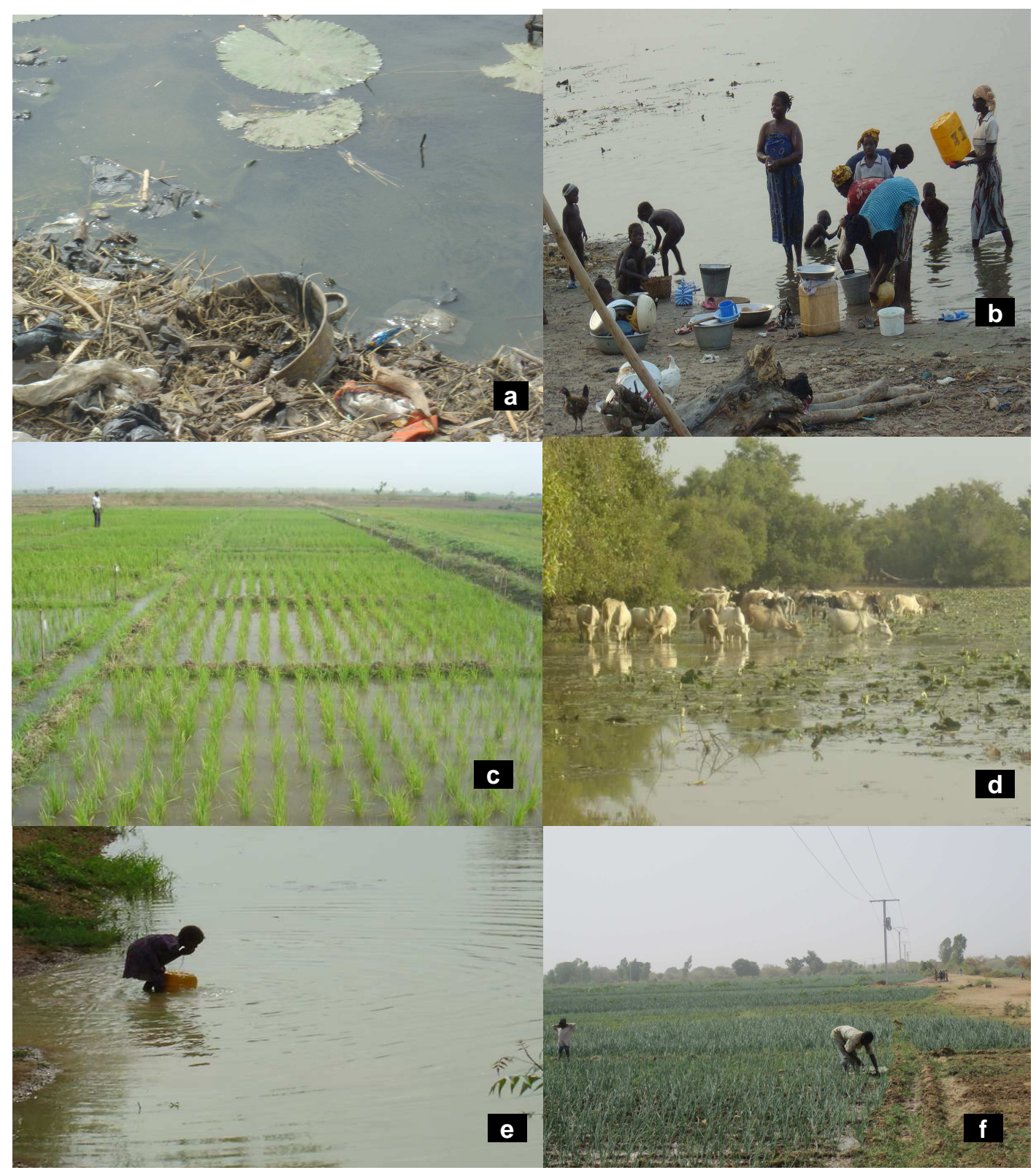

Figure 3 : Quelques principaux usages et fonctions des eaux de surface au niveau des points de contrôle dans la vallée du Sourou en période de forte demande. (a) déjection d'ordures et d'eaux usées à Toma-île aval ; (b) activités ménagères et récréatives à Toma-île amont ; (c) riziculture sur les berges à Débé aval; (d) abreuvage d'animaux au point de contrôle du Gana en fin de saison pluvieuse ; (e) approvisionnement en eau de boisson à tous les points de contrôle ; (f) cultures maraîchères particulièrement à Débé aval et Di aval. 
Tableau 1: Principaux usages de l'eau sur les sites des points de contrôle.

\begin{tabular}{|c|c|c|}
\hline Site & Cours d'eau & Usage de l'eau \\
\hline Léri (1) & Mouhoun & Pêche principalement \\
\hline Léri(2) & Mouhoun-Sourou & Pêche principalement \\
\hline Bissan & Sourou & $\begin{array}{l}\text { Pêche, spéculations agricoles, abreuvement } \\
\text { d'animaux, eau de boisson, activités recréationnelles. }\end{array}$ \\
\hline Yaran aval & Sourou & $\begin{array}{l}\text { Pêche, riziculture et cultures de contre saison, } \\
\text { abreuvement d'animaux en période de basses eaux. }\end{array}$ \\
\hline Débé aval & $\begin{array}{l}\text { Débé (affluent } \\
\text { Sourou) }\end{array}$ & $\begin{array}{l}\text { Riziculture, cultures maraîchères, lessives, } \\
\text { abreuvement d'animaux. }\end{array}$ \\
\hline Débé amont & $\begin{array}{l}\text { Débé (affluent } \\
\text { Sourou) }\end{array}$ & $\begin{array}{l}\text { Pêche, baignade d'hippopotames, abreuvement } \\
\text { d'animaux, station d'amenée du canal principal. }\end{array}$ \\
\hline $\begin{array}{l}\text { Toma-île } \\
\text { amont }\end{array}$ & Sourou & $\begin{array}{l}\text { Pêche, eau de boisson humaine, activités ménagères et } \\
\text { récréatives, abreuvement d'animaux (basses eaux). }\end{array}$ \\
\hline $\begin{array}{l}\text { Toma-île } \\
\text { aval }\end{array}$ & Sourou & $\begin{array}{l}\text { Pêche, activités ménagères et récréatives, déjections } \\
\text { d'ordures domestiques et d'eaux usées, abreuvement } \\
\text { d'animaux (basses eaux). }\end{array}$ \\
\hline Di aval & Sourou & $\begin{array}{l}\text { Pêche, abreuvage d'animaux, activités ménagères et } \\
\text { récréatives, spéculations agricoles et cultures } \\
\text { maraîchères sur les berges. }\end{array}$ \\
\hline Naré & Gana (affluent Débé) & $\begin{array}{l}\text { Abreuvage d'animaux, eau de boisson humaine, } \\
\text { activités domestiques et récréatives. }\end{array}$ \\
\hline
\end{tabular}

Tableau 2: Limite de classes des paramètres physico-chimiques et bactériologiques de la qualité des eaux de surface. (SEQ-Eau, MEDD et Agences de l'Eau 2003, France).

\begin{tabular}{lccccc}
\hline Qualité / Paramètre & Très Bonne & Bonne & Moyenne & Mauvaise & Très Mauvaise \\
\hline $\mathbf{P O}_{4}{ }^{2-}(\mathrm{mg} / \mathrm{L})$ & $0-0,1$ & $0,1-0,5$ & $0,5-1$ & $1-2$ & $>2$ \\
$\mathbf{N O}_{3}{ }^{-}(\mathrm{mg} / \mathrm{L})$ & $0-2$ & $2-10$ & $10-25$ & $25-50$ & $>50$ \\
$\mathbf{N H}_{4}{ }^{-}(\mathrm{mg} / \mathrm{L})$ & $0-0,1$ & $0,1-0,5$ & $0,5-2$ & $2-5$ & $>5$ \\
\hline $\begin{array}{c}\text { Escherichia coli } \\
(\mathrm{n} / 100 \mathrm{ml})\end{array}$ & 0 & $1-20$ & $21-200$ & $201-2000$ & $>2000$ \\
\hline $\begin{array}{c}\text { Coliformes fécaux } \\
(\mathrm{n} / 100 \mathrm{ml})\end{array}$ & 0 & $1-20$ & $21-200$ & $201-2000$ & $>2000$ \\
\hline
\end{tabular}




\section{DIANOU et al. / Int. J. Biol. Chem. Sci. 5(4): 1571-1589, 2011}

Tableau 3: Analyse de variance des paramètres physicochimiques des eaux en fonction du site et de la période d'échantillonnage.

\begin{tabular}{|c|c|c|c|c|c|c|c|c|c|c|c|c|c|c|c|c|c|c|c|c|c|c|c|}
\hline \multirow[t]{2}{*}{$\begin{array}{l}\text { Source de } \\
\text { variation }\end{array}$} & \multirow[t]{2}{*}{ ddl } & \multicolumn{2}{|c|}{$\underset{\left(\mathrm{mg} \mathrm{NH}_{4}^{+} / \mathrm{L}\right)}{\text { Ammonium }}$} & \multicolumn{2}{|c|}{$\begin{array}{c}\text { Conductivité } \\
(\mu \mathrm{S} / \mathrm{cm})\end{array}$} & \multicolumn{2}{|l|}{$\begin{array}{l}\text { DCO } \\
\left(\mathrm{mg} \mathrm{O}_{2} / \mathrm{L}\right)\end{array}$} & \multicolumn{2}{|c|}{$\begin{array}{c}\text { MS } \\
(\mathrm{mg} / \mathrm{L})\end{array}$} & \multicolumn{2}{|c|}{$\begin{array}{l}\text { Nitrates (mg } \\
\left.\mathrm{N}-\mathrm{NO}_{3}^{-} / \mathrm{L}\right)\end{array}$} & \multicolumn{2}{|c|}{$\begin{array}{c}\text { Nitrites } \\
\left(\mathrm{mg} \mathrm{NO}_{2} / \mathrm{L}\right)\end{array}$} & \multicolumn{2}{|c|}{$\begin{array}{l}\text { Ortho- } \\
\text { phosphate } \\
\left(\mathrm{mg} \mathrm{PO}_{4}{ }^{3-} / \mathrm{L}\right)\end{array}$} & \multicolumn{2}{|c|}{ pH } & \multicolumn{2}{|c|}{$\begin{array}{c}\mathbf{O}_{2} \text { dissous } \\
\left(\mathrm{mg} \mathrm{O}_{2} / \mathrm{L}\right)\end{array}$} & \multicolumn{2}{|c|}{$\begin{array}{l}\mathbf{O}_{2} \text { dissous } \\
\text { (saturation:\%) }\end{array}$} & \multicolumn{2}{|l|}{$\begin{array}{r}\text { Turbidité } \\
\text { (NTU) }\end{array}$} \\
\hline & & $\mathrm{CM}$ & $\mathrm{F}$ & $\mathrm{CM}$ & $\mathrm{F}$ & $\mathrm{CM}$ & F & $\mathrm{CM}$ & $\mathrm{F}$ & $\mathrm{CM}$ & F & $\mathrm{CM}$ & F & $\mathrm{CM}$ & $\mathrm{F}$ & $\mathrm{CM}$ & F & $\mathrm{CM}$ & F & $\mathrm{CM}$ & F & $\mathrm{CM}$ & $\mathrm{F}$ \\
\hline Période & 2 & 3,349 & $\mathrm{~ns}$ & 168097,87 & ** & 73290,2 & * & 164431,4 & * & 67,79 & ** & 0,02 & $* *$ & 0,17 & $* *$ & 14,86 & $* *$ & 5,33 & * & 1172,04 & * & 26602,52 & * \\
\hline Site & 9 & 31,81 & $* *$ & 1821,53 & $\mathrm{~ns}$ & 59225,35 & $* *$ & 116292,47 & $* *$ & 14,52 & $* *$ & 0,01 & $* *$ & 0,11 & $* *$ & 2,60 & $* *$ & 11,18 & $* *$ & 1787,40 & ** & 7039,50 & ** \\
\hline $\begin{array}{c}\text { Période } \\
\mathbf{x} \\
\text { Site }\end{array}$ & 28 & 16,98 & $* *$ & 16596,75 & $* *$ & 51269,14 & $* *$ & 118657,97 & $* *$ & 12,93 & $* *$ & 0,01 & $* *$ & 0,11 & $* *$ & 1,80 & $* *$ & 5,19 & $* *$ & 838,66 & $* *$ & 39496,84 & $* *$ \\
\hline
\end{tabular}

MS = matières en suspensions $; \mathbf{C M}=$ carrés moyens $; *=$ significatif au seuil de $5 \% ; \quad * *=$ significatif au seuil de $1 \% ; \mathbf{n s}=$ non significatif. 
D. DIANOU et al. / Int. J. Biol. Chem. Sci. 5(4): 1571-1589, 2011

Tableau 4 : Paramètres physico-chimiques des échantillons d'eau des rivières Mouhoun, Sourou et affluents dans le bassin du Sourou lors des périodes d'échantillonnage 1 (Novembre 2007), 2 (Février 2008) et 3 (Juin 2008) (résultats moyens de 3 échantillons).

\begin{tabular}{|c|c|c|c|c|c|c|c|c|c|c|c|c|}
\hline $\begin{array}{l}\text { Site } \\
\text { (cours d'eau) }\end{array}$ & Période & $\begin{array}{l}\text { Ammonium } \\
\left(\mathrm{mg} \mathrm{NH}_{4}{ }^{+} / \mathrm{L}\right)\end{array}$ & $\begin{array}{c}\text { Conductivité } \\
(\mu \mathrm{S} / \mathrm{cm})\end{array}$ & $\begin{array}{c}\text { DCO } \\
\left(\mathrm{mg} \mathrm{O}_{2} / \mathrm{L}\right)\end{array}$ & $\begin{array}{c}\text { MS } \\
(\mathrm{mg} / \mathrm{L})\end{array}$ & 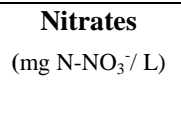 & $\begin{array}{c}\text { Nitrites } \\
\left(\mathrm{mg} \mathrm{NO}_{2}^{-} / \mathrm{L}\right)\end{array}$ & $\begin{array}{c}\text { Orthophos } \\
\text { phates } \\
\left(\mathrm{mg} \mathrm{PO}_{4}{ }^{3-} / \mathrm{L}\right)\end{array}$ & pH & $\begin{array}{c}\mathrm{O}_{2} \\
\text { dissous } \\
\left(\mathrm{mg} \mathrm{O}_{2} / \mathrm{L}\right)\end{array}$ & $\begin{array}{c}\mathrm{O}_{2} \text { dissous } \\
\text { (saturation:\%) }\end{array}$ & $\begin{array}{c}\text { Turbidité } \\
\text { (NTU) }\end{array}$ \\
\hline Toma-île & 1 & $0,30^{\mathrm{c}}$ & $116^{\mathrm{m}}$ & $15^{\mathrm{h}}$ & $10^{\mathrm{e}}$ & $0,5^{\text {ef }}$ & $0,004^{\mathrm{e}}$ & $0,23^{\mathrm{ef}}$ & $7,4^{\mathrm{fgh}}$ & $6,1^{i}$ & $77,7^{\mathrm{n}}$ & $8,0^{k}$ \\
\hline amont & 2 & $0,24^{\mathrm{c}}$ & $177^{\mathrm{i}}$ & $26^{\mathrm{g}}$ & $0^{\mathrm{f}}$ & $3,0^{\mathrm{cd}}$ & $0,011^{\mathrm{e}}$ & $0,21^{\mathrm{fg}}$ & $7,0^{\mathrm{ijk}}$ & $6,4^{\mathrm{h}}$ & $82,6^{1}$ & $3,6^{\mathrm{m}}$ \\
\hline \multirow[t]{2}{*}{ (Sourou) } & 3 & nd & $301^{\mathrm{b}}$ & nd & nd & $3,0^{\mathrm{cd}}$ & $0,002^{\mathrm{e}}$ & $0,17^{\mathrm{g}}$ & $8,5^{\mathrm{bc}}$ & $5,5^{\mathrm{j}}$ & $75,0^{\mathrm{p}}$ & $157,0^{\mathrm{b}}$ \\
\hline & 1 & $0,27^{\mathrm{c}}$ & $117^{\mathrm{m}}$ & $24^{\mathrm{g}}$ & $2^{f}$ & $0,4^{\mathrm{ef}}$ & $0,002^{\mathrm{e}}$ & $0,25^{\mathrm{de}}$ & $7,2^{\mathrm{hi}}$ & $6,4^{\mathrm{h}}$ & $82,0^{1}$ & $6,0^{\mathrm{klm}}$ \\
\hline Toma-île aval & 2 & $0,26^{\mathrm{c}}$ & $178^{\mathrm{i}}$ & $90^{\mathrm{b}}$ & $0^{\mathrm{f}}$ & $4,6^{\mathrm{b}}$ & $0,008^{\mathrm{e}}$ & $0,09^{\mathrm{h}}$ & $6,9^{\mathrm{jk}}$ & $6,5^{\mathrm{gh}}$ & $84,2^{\mathrm{k}}$ & $3,4^{\mathrm{m}}$ \\
\hline \multirow[t]{2}{*}{ (Sourou) } & 3 & nd & $289^{\mathrm{cd}}$ & nd & nd & $5,6^{\mathrm{b}}$ & $0,010^{\mathrm{e}}$ & $0,02^{\mathrm{i}}$ & $8,4^{\mathrm{bc}}$ & $7,7^{\mathrm{b}}$ & $105,0^{\mathrm{b}}$ & $28,0^{\mathrm{f}}$ \\
\hline & 1 & $0,15^{\mathrm{c}}$ & $117^{\mathrm{m}}$ & $15^{\mathrm{h}}$ & $10^{\mathrm{e}}$ & $0,4^{\mathrm{ef}}$ & $0,002^{\mathrm{e}}$ & $0,20^{\mathrm{fg}}$ & $7,05^{\mathrm{ij}}$ & $6,4^{\mathrm{h}}$ & $82,0^{1}$ & $0,15^{\mathrm{n}}$ \\
\hline Di aval & 2 & $0,06^{\mathrm{c}}$ & $180^{\mathrm{i}}$ & $23^{\mathrm{g}}$ & $0^{\mathrm{f}}$ & $4,2^{\mathrm{bc}}$ & $0,002^{\mathrm{e}}$ & $0,13^{\mathrm{h}}$ & $7,2^{\text {hi }}$ & $6,8^{\mathrm{efg}}$ & $88,2^{\mathrm{i}}$ & $6,0^{\mathrm{klm}}$ \\
\hline \multirow[t]{2}{*}{ (Sourou) } & 3 & nd & $291^{\mathrm{bc}}$ & nd & nd & $9,2^{\mathrm{a}}$ & $0,030^{\mathrm{de}}$ & $0,20^{\mathrm{fg}}$ & $8,4^{\mathrm{bc}}$ & $8.3^{\mathrm{a}}$ & $113,0^{\mathrm{a}}$ & $23,0^{\mathrm{g}}$ \\
\hline & 1 & $0,21^{\mathrm{c}}$ & $113^{\mathrm{m}}$ & $11^{i}$ & $20^{\mathrm{d}}$ & $0,3^{\text {ef }}$ & $0,002^{\mathrm{e}}$ & $0,12^{\mathrm{h}}$ & $7,3^{\text {gh }}$ & $5,1^{\mathrm{k}}$ & $64,8^{\mathrm{s}}$ & $7,0^{\mathrm{KI}}$ \\
\hline Yaran & 2 & $0,02^{\mathrm{c}}$ & $162^{\mathrm{j}}$ & $25^{\mathrm{g}}$ & $0^{\mathrm{f}}$ & $2,1^{\mathrm{de}}$ & $0,029^{\mathrm{de}}$ & $0,65^{\mathrm{b}}$ & $7,5^{\mathrm{fg}}$ & $6,9^{\mathrm{ef}}$ & $89,3^{\text {hi }}$ & $3,8^{\mathrm{m}}$ \\
\hline \multirow[t]{2}{*}{ (Sourou) } & 3 & nd & $236^{\mathrm{f}}$ & nd & nd & $2,5^{\mathrm{d}}$ & $0,015^{\mathrm{e}}$ & $0,12^{\mathrm{h}}$ & $8,4^{\mathrm{bc}}$ & $6,9^{\mathrm{ef}}$ & $96,0^{\mathrm{d}}$ & $14,0^{\mathrm{i}}$ \\
\hline & 1 & $0,08^{\mathrm{c}}$ & $111^{\mathrm{m}}$ & $51^{\mathrm{e}}$ & $10^{\mathrm{e}}$ & $0,3^{\text {ef }}$ & $0,014^{\mathrm{e}}$ & $0,19^{\mathrm{fg}}$ & $7,4^{\mathrm{fgh}}$ & $7,7^{\mathrm{b}}$ & $94,0^{\mathrm{e}}$ & $5,0^{\operatorname{lm}}$ \\
\hline Bissan & 2 & $0,09^{\mathrm{c}}$ & $149^{\mathrm{k}}$ & $16^{\mathrm{h}}$ & $0^{\mathrm{f}}$ & $2,0^{\mathrm{de}}$ & $0,067^{\mathrm{c}}$ & $0,02^{\mathrm{i}}$ & $8,1^{\mathrm{d}}$ & $6,8^{\text {efg }}$ & $89,5^{\mathrm{h}}$ & $5,0^{\operatorname{lm}}$ \\
\hline (Sourou) & 3 & nd & $216^{\mathrm{g}}$ & nd & nd & $2,8^{\mathrm{cd}}$ & $0,018^{\mathrm{e}}$ & $0,04^{i}$ & $8,6^{\mathrm{b}}$ & $6,5^{\mathrm{gh}}$ & $87,0^{\mathrm{j}}$ & $12,0^{\mathrm{ij}}$ \\
\hline
\end{tabular}


D. DIANOU et al. / Int. J. Biol. Chem. Sci. 5(4): 1571-1589, 2011

\begin{tabular}{|c|c|c|c|c|c|c|c|c|c|c|c|c|}
\hline & 1 & $0,13^{\mathrm{c}}$ & $115^{\mathrm{m}}$ & $4^{j}$ & $30^{c}$ & $0,5^{\mathrm{ef}}$ & $0,003^{\mathrm{e}}$ & $0,27^{\mathrm{d}}$ & $7,4^{\text {fgh }}$ & $7,4^{\mathrm{c}}$ & $91,0^{g}$ & $21,0^{\mathrm{h}}$ \\
\hline Léri (2) & 2 & $0,02^{\mathrm{c}}$ & $204^{\mathrm{h}}$ & $11^{\mathrm{i}}$ & $0^{\mathrm{f}}$ & $5,6^{\mathrm{b}}$ & $0,020^{\mathrm{e}}$ & $0,09^{\mathrm{h}}$ & $7,6^{\mathrm{f}}$ & $6,8^{\mathrm{efg}}$ & $88,3^{\mathrm{i}}$ & $5,0^{\mathrm{lm}}$ \\
\hline (Sourou) & 3 & nd & $216^{\mathrm{g}}$ & nd & nd- & $2,8^{\mathrm{cd}}$ & $0,113^{\mathrm{b}}$ & $0,09^{\mathrm{h}}$ & $8,6^{\mathrm{b}}$ & $5,2^{\mathrm{k}}$ & $71,0^{r}$ & $28,0^{\mathrm{f}}$ \\
\hline Léri (1) & 1 & $0,13^{\mathrm{c}}$ & $134^{1}$ & $3^{j}$ & $10^{\mathrm{e}}$ & $2,0^{\mathrm{de}}$ & $0,007^{\mathrm{e}}$ & $0,34^{\mathrm{c}}$ & $7,4^{\mathrm{fgh}}$ & $7,3^{\text {cd }}$ & $98,0^{c}$ & $14,0^{i}$ \\
\hline (Mouhoun- & 2 & $0,02^{\mathrm{c}}$ & $207^{\mathrm{h}}$ & $10^{\mathrm{i}}$ & $0^{\mathrm{f}}$ & $1,9^{\mathrm{def}}$ & $0,010^{\mathrm{e}}$ & $0,10^{\mathrm{h}}$ & $7,8^{\mathrm{e}}$ & $6,9^{\text {ef }}$ & $89,8^{\mathrm{h}}$ & $4,0^{\mathrm{m}}$ \\
\hline Sourou) & 3 & nd & $265^{\mathrm{e}}$ & nd & nd & $2,4^{\mathrm{d}}$ & $0,368^{\mathrm{a}}$ & $0,11^{\mathrm{h}}$ & $8,9^{\mathrm{a}}$ & $5,6^{\mathrm{j}}$ & $76,0^{\circ}$ & $11,{ }^{0 \mathrm{j}}$ \\
\hline Débé amont & 1 & $0,22^{\mathrm{c}}$ & $116^{\mathrm{m}}$ & $22^{g}$ & $20^{\mathrm{d}}$ & $0,3^{\text {ef }}$ & $0,002^{\mathrm{e}}$ & $0,20^{\mathrm{fg}}$ & $6,8^{k}$ & $5,0^{\mathrm{k}}$ & $74,5^{\mathrm{p}}$ & $7,0^{\mathrm{KI}}$ \\
\hline (affluent & 2 & $0,43^{\mathrm{c}}$ & $173^{\mathrm{i}}$ & $44^{\mathrm{f}}$ & $0,1^{\mathrm{f}}$ & $1,6^{\mathrm{def}}$ & $0,035^{\mathrm{de}}$ & $0,33^{\mathrm{c}}$ & $7,9^{\mathrm{de}}$ & $7,1^{\text {de }}$ & $92,2^{\mathrm{f}}$ & $10,7^{\mathrm{j}}$ \\
\hline Sourou) & 3 & nd & $281^{\mathrm{d}}$ & nd & nd & $0,4^{\mathrm{ef}}$ & $0,002^{\mathrm{e}}$ & $0,21^{\mathrm{fg}}$ & $8,3^{\mathrm{c}}$ & $6,7^{\text {fgh }}$ & $91,0^{\mathrm{g}}$ & $89,0^{\mathrm{c}}$ \\
\hline Débé aval & 1 & $0,25^{\mathrm{c}}$ & $119^{\mathrm{m}}$ & $18^{\mathrm{h}}$ & $40^{\mathrm{b}}$ & $0,6^{\mathrm{ef}}$ & $0,005^{\mathrm{e}}$ & $0,28^{\mathrm{d}}$ & $7,4^{\mathrm{fgh}}$ & $5,7^{j}$ & $82,0^{1}$ & $8,0^{\mathrm{k}}$ \\
\hline (affluent & 2 & $0,35^{\mathrm{c}}$ & $177^{\mathrm{i}}$ & $85^{\mathrm{c}}$ & $0,2^{\mathrm{f}}$ & $4,7^{\mathrm{b}}$ & $0,050^{\mathrm{cd}}$ & $0,09^{\mathrm{h}}$ & $7,9^{\mathrm{de}}$ & $5,5^{\mathrm{j}}$ & $72,0^{\mathrm{q}}$ & $6,0^{\mathrm{klm}}$ \\
\hline Sourou) & 3 & nd & $285^{\mathrm{cd}}$ & nd & nd & $2,3^{\mathrm{d}}$ & $0,003^{\mathrm{e}}$ & $0,09^{\mathrm{h}}$ & $8,4^{\mathrm{bc}}$ & $5,8^{\mathrm{j}}$ & $79,0^{\mathrm{m}}$ & $40,0^{\mathrm{e}}$ \\
\hline Gana/Naré & 1 & $5,00^{b}$ & $52^{\mathrm{n}}$ & $71^{d}$ & $896^{\mathrm{a}}$ & $0,3^{\text {ef }}$ & $0,002^{\mathrm{e}}$ & $0,10^{h}$ & $6,2^{1}$ & $1,1^{1}$ & $16,6^{t}$ & $614,0^{\mathrm{a}}$ \\
\hline (affluent & 2 & $9,90^{\mathrm{a}}$ & $353^{\mathrm{a}}$ & $603^{a}$ & $0,7^{\mathrm{f}}$ & $0,1^{\mathrm{f}}$ & $0,018^{\mathrm{e}}$ & $0,98^{\mathrm{a}}$ & $5,5^{\mathrm{m}}$ & nd & nd & $50,0^{\mathrm{d}^{* * *}}$ \\
\hline Débé) & $3 *$ & nd & nd & nd & nd & nd & nd & nd & nd & nd & nd & nd \\
\hline Normes OMS & & 1,50 & $50-150$ & - & - & 11,4 & 3 & 5 & $6,5-8,5$ & - & - & $\leq 5$ \\
\hline
\end{tabular}

MS : matières en suspensions ; nd : non déterminé ; $*$ : cours d'eau asséché ; ${ }^{* *}$ :> 7000 NTU en 2011

Les valeurs qui ont une lettre en commun dans une colonne ne sont pas significativement différentes selon le test de Newman-Keuls au seuil de $5 \%$. 
Tableau 5 : Analyse de variance des paramètres bactériologiques en fonction du site et de la période d'échantillonnage.

\begin{tabular}{|c|c|c|c|c|c|c|c|}
\hline \multirow[t]{2}{*}{$\begin{array}{l}\text { Source de } \\
\text { variation }\end{array}$} & \multirow[t]{2}{*}{ ddl } & \multicolumn{3}{|c|}{$\begin{array}{c}\begin{array}{c}\text { Escherichia coli } \\
(\mathrm{n} / 100 \mathrm{ml})\end{array} \\
\end{array}$} & \multicolumn{3}{|c|}{$\begin{array}{c}\text { Coliformes fécaux } \\
(\mathrm{n} / 100 \mathrm{ml})\end{array}$} \\
\hline & & CM & $\mathbf{p}$ & $\overline{\mathbf{F}}$ & $\mathbf{C M}$ & $\mathbf{p}$ & $\mathbf{F}$ \\
\hline Période & 2 & 9320142,460 & 0,184 & $\mathrm{~ns}$ & 3429281,009 & 0,696 & $\mathrm{~ns}$ \\
\hline Site & 9 & 42096193,121 & $<0,0001$ & $* *$ & 82951510,588 & $<0,0001$ & $* *$ \\
\hline Site x Période & 28 & 16847573,342 & $<0,0001$ & $* *$ & 28508314,149 & $<0,0001$ & ** \\
\hline
\end{tabular}

$\mathbf{C M}=$ Carrés moyens $; * *=$ significatif au seuil de $1 \% ; \mathbf{n s}=$ non significatif au seuil de $5 \%$

\section{DISCUSSION \\ Qualité des eaux}

Les résultats analytiques présentés dans cette étude représentent les déterminations physico-chimiques, minérales, organiques et bactériologiques analysées en référence à différentes normes en vigueur. Les prélèvements effectués dans le cadre des campagnes de mesure sont de type instantané; ils ne sont ainsi que le reflet de la qualité de l'eau à un moment donné et montrent l'influence locale des sources de pollution (activités domestiques, pêche, élevage et activités agricoles).

\section{Qualité physico-chimique}

Au plan physico-chimique les résultats de cette étude indiquent que les valeurs observées pour les différents paramètres mesurés sont en lien avec la période d'échantillonnage et le point de contrôle, excepté l'ammonium pour lequel la période ne semble pas avoir d'effet (Tableau 3). Globalement les plans d'eau des rivières Mouhoun, Sourou, Débé et Gana qui irriguent la vallée sont de bonne qualité en termes d'aptitude à présenter des potentialités biologiques et pour divers usages au regard des normes du système d'évaluation de l'eau des cours d'eau (SQE-Eau, 2003 ; Tableau 2). Toutefois, les plans d'eau au niveau du Gana,
Toma-île aval, Débé aval et Débé amont présentent des pollutions prononcées pendant la période sèche avec respectivement des DCO de 603, 90, 85 et $44 \mathrm{mg} \mathrm{O} / \mathrm{L}$ (Tableau 4). La période sèche correspond à la période de basses eaux et de fortes pressions sur les eaux de surface dans la vallée. Le site du Gana connait en cette période une intensification de l'abreuvage d'animaux (Tableau 1, Figure 3d). Durant cette période sèche le site de Débé, particulièrement Débé amont, est le lieu de prédilection pour les baignades et autres activités des hippopotames (Tableau 1). Quant au village de Toma-île, logé sur la rivière Sourou, l'aval du village reçoit en cette période les ordures ménagères, les eaux usées et autres déjections (Tableau 1, Figure 3a). Les déjections diverses au niveau de ces points de contrôle créent des conditions propices à une activité biologique plus ou moins prononcée et expliqueraient en partie les valeurs de DCO observées sur ces sites en cette période de l'année. Des teneurs élevées en ammonium sont particulièrement observées au point de contrôle du Gana en fin

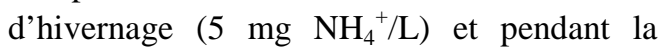
période sèche $\left(9,9 \mathrm{mg} \mathrm{NH}_{4}{ }^{+} / \mathrm{L}\right)$ (Tableau 4). $\mathrm{La}$ fréquentation intensive d'animaux et les déjections animales récentes observées au moment de l'échantillonnage sur ce site (Figure 3d) pourraient expliquer ces teneurs. 
Tableau 6 : Paramètres bactériologiques des échantillons d'eau des rivières Mouhoun, Sourou et affluents dans le bassin du Sourou lors des périodes d'échantillonnage 1 (Novembre 2007), 2 (Février 2008) et 3 (Juin 2008) (résultats moyens de 3 échantillons).

\begin{tabular}{|c|c|c|c|}
\hline $\begin{array}{l}\text { Site } \\
\text { (cours d'eau) }\end{array}$ & $\begin{array}{c}\text { Période } \\
\text { d'échantillonnage }\end{array}$ & $\begin{array}{c}\text { E. coli } \\
(\mathrm{n} / 100 \mathrm{ml})\end{array}$ & $\begin{array}{c}\text { Coliformes } \\
\text { fécaux } \\
(\mathrm{n} / 100 \mathrm{ml})\end{array}$ \\
\hline & 1 & $27^{\mathrm{fg}}$ & $700^{f}$ \\
\hline Toma-île amont & 2 & $62^{\mathrm{fg}}$ & $200^{\mathrm{jklmn}}$ \\
\hline \multirow[t]{2}{*}{ (Sourou) } & 3 & $113^{\mathrm{fg}}$ & $320^{\text {ghijk }}$ \\
\hline & 1 & $00^{\mathrm{g}}$ & $00^{\mathrm{p}}$ \\
\hline Toma-île aval & 2 & $393^{\mathrm{d}}$ & $1000^{\mathrm{e}}$ \\
\hline \multirow[t]{2}{*}{ (Sourou) } & 3 & $1260^{c}$ & $5000^{c}$ \\
\hline & 1 & $00^{\mathrm{g}}$ & $22^{\mathrm{op}}$ \\
\hline Di aval & 2 & $33^{\mathrm{fg}}$ & $120^{\mathrm{mnop}}$ \\
\hline \multirow[t]{2}{*}{ (Sourou) } & 3 & $47^{\mathrm{fg}}$ & $187^{\mathrm{klmno}}$ \\
\hline & 1 & $39^{\mathrm{fg}}$ & $372^{\text {ghi }}$ \\
\hline Yaran & 2 & $60^{\mathrm{fg}}$ & $107^{\text {mnop }}$ \\
\hline \multirow[t]{2}{*}{ (Sourou) } & 3 & $00^{\mathrm{g}}$ & $340^{\text {ghij }}$ \\
\hline & 1 & $00^{\mathrm{g}}$ & $234^{\mathrm{ijklm}}$ \\
\hline Bissan & 2 & $00^{\mathrm{g}}$ & $27^{\text {op }}$ \\
\hline \multirow[t]{2}{*}{ (Sourou) } & 3 & $07^{\mathrm{g}}$ & $20^{\mathrm{op}}$ \\
\hline & 1 & $84^{\mathrm{fg}}$ & $434^{\mathrm{g}}$ \\
\hline Léri (2) & 2 & $07^{\mathrm{g}}$ & $87^{\text {mnop }}$ \\
\hline \multirow[t]{2}{*}{ (Sourou) } & 3 & $120^{\mathrm{fg}}$ & $1150^{\mathrm{d}}$ \\
\hline & 1 & $143^{\mathrm{f}}$ & $606^{\mathrm{f}}$ \\
\hline Léri (1) & 2 & $40^{\mathrm{fg}}$ & $53^{\text {nop }}$ \\
\hline \multirow[t]{2}{*}{ (Mouhoun-Sourou) } & 3 & $13^{\mathrm{g}}$ & $73^{\text {nop }}$ \\
\hline & 1 & $00^{\mathrm{g}}$ & $00^{\mathrm{p}}$ \\
\hline Débé amont & 2 & $127^{\mathrm{fg}}$ & $273^{\text {hilkl }}$ \\
\hline \multirow[t]{2}{*}{ (affluent Sourou) } & 3 & $247^{\mathrm{e}}$ & $387^{\text {gh }}$ \\
\hline & 1 & $28^{\mathrm{fg}}$ & $684^{\mathrm{f}}$ \\
\hline Débé aval & 2 & $40^{\mathrm{fg}}$ & $134^{\operatorname{lmnop}}$ \\
\hline \multirow[t]{2}{*}{ (affluent Sourou) } & 3 & $67^{\mathrm{fg}}$ & $28^{\mathrm{op}}$ \\
\hline & 1 & $4445^{b}$ & $10856^{b}$ \\
\hline Gana/Naré & 2 & $12200^{\mathrm{a}}$ & $12800^{\mathrm{a}}$ \\
\hline (affluent Débé) & $3 *$ & nd & nd \\
\hline OMS (2004), eau de & isson & 0 & 0 \\
\hline
\end{tabular}

nd: non déterminé ; * : cours d'eau asséché; Les valeurs ayant une lettre en commun dans une colonne ne sont pas significativement différentes selon le test de Newman-Keuls au seuil de $5 \%$. 
En outre, pour la presque totalité des points de contrôle, la turbidité des plans d'eau est plus prononcée en début d'hivernage (Juin), excepté celui du Gana pour lequel aucun échantillon n'a pu être prélevé du fait de l'assèchement de ce site en cette période (période 3, Tableau 4). Toutefois, contrastant nos résultats de 2008 pour ce paramètre, nous avons enregistré une turbidité de plus 7000 NTU pour la même période sur ce site en 2011 (Kaboré, 2011), ce qui laisse entrevoir des valeurs encore plus élevées dès les premières eaux de remplissage en saison hivernale et ce en accord avec l'évolution observée pour ce paramètre sur les autres sites. Nos résultats s'accordent sur ce point avec ceux obtenus par Somé et al. (2008) pour les eaux des réservoirs de Loumbila et Mogtédo au Burkina Faso, qui ont effectivement souligné que la turbidité des eaux de surface observée en début de saison pluvieuse est intimement liée à l'intensité des pluies et au ruissellement des eaux qui s'ensuit sur des sols agricoles non encore couverts par la végétation.

La valeur la plus élevée pour la conductivité électrique est observée sur le site du Gana en période sèche $(353 \mu \mathrm{S} / \mathrm{cm})$. Les déjections animales entrainant un apport de matières organiques dans les eaux, du fait de la fréquentation intensive d'animaux à ce point de contrôle en cette période peut expliquer la minéralisation légèrement plus importante et corrélativement le bas $\mathrm{pH}$ des eaux $(5,5)$ observés sur ce site (Tableau 4) comme l'ont également souligné Somé et al. (2008).

Les concentrations les plus élevées en nitrates sont observées sur les sites de Di aval, Toma-île aval et Débé aval. Au niveau du village insulaire de Toma-île, les déjections d'ordures ménagères, eaux usées et autres dans la partie jouxtant l'aval du cours d'eau (Figure 3a) en période de basses eaux peuvent expliquer les concentrations en nitrates observées dans les eaux de ce point de contrôle. Par ailleurs, la riziculture irriguée (Figure 3c) et la production d'oignon (Figure 3e) au voisinage du cours d'eau à Débé aval et les productions de tomate, oignons et autres cultures maraîchères aux abords du point de contrôle à Di aval sont les principales activités de contre-saison sur ces sites (Traoré, 2003). Pour ces cultures, les producteurs utilisent des engrais minéraux, notamment du NPK et de l'urée. La recherche de bon rendement conduit le plus souvent à une utilisation importante de ces engrais parfois au-delà des prescriptions techniques. Une production intensive de tomate est particulièrement développée aux abords du cours d'eau au niveau de Di aval au mois de Mai. A cette période, le bas niveau du cours d'eau conduit à l'installation des parcelles presque dans le lit du réservoir et à l'utilisation de grosses motopompes pour irriguer les parcelles avec souvent pour conséquences un trop plein d'eau contenant les minéraux qui retourne à la rivière. Ces pratiques culturales, en plus du drainage des minéraux à travers les eaux de ruissellement en début de saison pluvieuse, peuvent justifier les concentrations plus élevées en nitrates dans les eaux de ces points de contrôle, en particulier à Di aval. Des observations similaires ont été faites par Somé et al. (2008) au niveau du réservoir de Mogtédo au Burkina Faso où l'oignon est la principale spéculation en saison sèche. En outre à Di aval, la période de forte turbidité coïncide avec la concentration la plus élevée en nitrates (Tableau 4). Ce résultat s'accorde avec ceux obtenus par Pouilleute (1996), Turpin et al. (2003), Plean et al. (2004) et Biney et al. (2005) qui ont conclu à la pollution diffuse de l'eau de surface par les activités agricoles. Les turbidités les plus élevées enregistrées au niveau de Toma-île amont (157 NTU) et Débé amont (89 NTU) durant la période de début de saison pluvieuse (période qui enregistre le plus bas niveau des eaux de la rivière Sourou) 
sont essentiellement liées aux activités ménagères et récréatives des populations de Toma-île (Figure 3b) et aux baignades d'hippopotames aux alentours de ce point de contrôle.

En considérant les niveaux de référence de la qualité des eaux de surface tels que définis par Nesbet et Vernaux (1970), les teneurs en nitrates et phosphates enregistrées présentent un risque pour l'induction de l'eutrophisation (phosphates $\geq 0,01 \mathrm{mg} / \mathrm{L}$ et nitrates $\geq 0,3 \mathrm{mg} / \mathrm{L}$ ) dans les eaux de l'ensemble des sites, en particulier dans celles de Di aval et du Gana (Figures 3a, 3d; Tableau 4).

$\mathrm{Au}$ regard des normes pour les eaux de boisson (WHO, 2004), les eaux des différents points de contrôle sont globalement impropres à la consommation humaine, en particulier en début de saison pluvieuse.

\section{Qualité bactériologique}

Des résultats d'analyse, il ressort clairement que sur un plan d'ensemble, les contaminations fécales observées sont plus ou moins prononcées en fonction des périodes de l'année. La période de Février à Juin correspond à une période de forte pression sur les eaux de la rivière Sourou et de ses affluents (Débé et Gana). De fortes concentrations sont observées particulièrement au point de contrôle du Gana en fin de saison pluvieuse et pendant la période sèche, respectivement pour Escherichia coli (4445 et 12200 UFC/100 ml) et les coliformes fécaux (10856 et 12800 UFC/100 ml). L'abreuvement intensif d'animaux qui à l'occasion s'y baignent et y défèquent et urinent crée les conditions propices à la prolifération de nombreux microorganismes entériques transformant ce point d'eau en un bouillon microbien en période sèche. Ces activités justifient les fortes pollutions microbiennes des eaux enregistrées sur ce site aux différentes périodes.

Une situation semblable est observée au niveau des points de contrôle avoisinant le village insulaire de Toma-île. Des concentrations de 1260 et 393 UFC/100 ml pour Escherichia coli et de 5000 et 1000 $\mathrm{UFC} / 100 \mathrm{ml}$ pour les coliformes fécaux sont révélées dans les eaux à Toma-île aval respectivement en début de saison pluvieuse et pendant la période sèche (Tableau 6). L'absence d'assainissement de base au niveau du village ( absence de latrines et de recyclage des déchets solides) conduit les populations du village à utiliser le côté du village en regard de l'aval du cours d'eau pour le dépôt d'ordures ménagères, l'évacuation des eaux usées des douches et autres selles des enfants pendant les périodes de basses eaux. Cette situation explique bien les fortes contaminations fécales trouvées dans les eaux des points de contrôle jouxtant le village en particulier au niveau de toma-île aval. La même situation prévaut au niveau des autres villages situés sur le cours d'eau et est particulièrement dommageable pour la santé des populations ainsi que nos investigations l'indiquent (Traoré, 2003 ; Dianou et al., 2003 et 2004; Poda et al., 2001, 2004 et 2006 ; Koukounary et al., 2007; Poda, 2007).

D’une manière générale, les eaux des petits réservoirs comme celui du Gana (qui fonctionne pratiquement comme un lac au niveau du point de contrôle) sont plus sensibles aux pollutions que celles des grands réservoirs (Sourou, Débé) caractérisés par une qualité différenciée des eaux d'un emplacement à un autre.

\section{Perspectives de développement durable dans la vallée du Sourou}

L'eau constitue un enjeu majeur pour le développement durable. Elle est étroitement liée à la santé, l'agriculture, l'énergie et la biodiversité. Comme des auteurs l'ont si bien 
souligné, sans une gestion efficace et efficiente des ressources en eau qui prenne en compte non seulement leur rareté mais également leur qualité, les sociétés deviennent minées par des conditions d'hygiène difficiles et des maladies hydriques, entravant par voie de conséquence leur développement socioéconomique (Phillips et al., 2006 ; Julien, 2006). Le Sommet Mondial du Développement Durable tenu à Johannesburg en 2002 appelle à développer la Gestion Intégrée des Ressources en Eau (GIRE) dans les pays en développement. Cette approche flexible qui permet de s'attaquer aux défis de l'eau et d'optimiser la contribution de l'eau dans le développement durable, encourage le développement et la gestion coordonnée de l'eau, des terres et des ressources connexes, en vue de maximiser de manière équitable, le bien-être économique et social sans compromettre la pérennité d'écosystèmes vitaux (Tec, 2005). Outre la gestion des ressources physiques, la GIRE implique la réforme des systèmes humains pour permettre aux individus de retirer le maximum de cette ressource (Kouam et al., 2006).

La présente étude qui vise à la mise en place d'un réseau de contrôle de la qualité des eaux de surface dans la vallée du Sourou se situe dans le cadre d'un contrat de rivière : le "Contrat de Rivière Sourou". Cette structure créée en 2003 sous l'initiative de la Convention pour la Promotion d'un Développement durable (COPROD) et du Département des Sciences et Gestion de l'Environnement de l'Université de Liège avec l'appui de la Région Wallonne de Belgique et de quatre communes Belges engagées dans le "Contrat de Rivière Semois", vise à asseoir une gestion consensuelle, partenariale et participative des multiples fonctions et usages de la rivière Sourou, de ses abords et des ressources en eau de son bassin en fonction des réalités locales. A travers une approche écosystémique qui intègre les aspects sociaux, culturels, économiques et sanitaires, vise également à positionner ce modèle en tant qu'instrument efficace et efficient de gestion intégrée des ressources en eau pour un développement durable dans la vallée du Sourou (Rosillon, 2004 et 2007 ; Rosillon et al., 2005 ; Rosillon et Bado, 2006). $\mathrm{Au}$ nombre des réalisations à ce jour du "Contrat de Rivière Sourou", on note l'organisation du bassin versant du Sourou en "Zones de Développement Durables", la mise en place de "Comités de Rivières", la réalisation de points d'eau potable, diverses formations et rencontres dans le cadre de la GIRE du bassin versant du Sourou, ... La mise en place d'un réseau performant de suivi de la qualité des eaux dans la vallée du Sourou constitue un des éléments essentiels d'aide à la prise de décisions pour préserver la qualité des ressources en eau et pour un développement harmonieux et durable dans la vallée. Ce dernier aspect est d'autant plus urgent si l'on prend en compte le fait qu'en dépit de la qualité médiocre des masses d'eau en certaines périodes, elles servent de source d'eau de boisson aux populations insulaires et riveraines avec pour conséquences une persistance et une amplification de maladies hydriques (Traoré 2003 ; Dianou et al., 2004; Poda et al., 2006; Koukounari et al., 2007). En ce sens, des actions ciblées devront s'attaquer entre autres aux points suivants :

- La prise en compte des aspects culturels et identitaires et des facteurs de cohésion sociale dans la gestion des ressources en eau ;

- L'implication des populations et de tous les acteurs concernés dans la gestion des ressources en eau de la vallée suivant un espace géographique bien défini ;

- L'approvisionnement en eau potable et la mise à disposition d'infrastructures d'assainissement de base (latrines) et de traitement des déchets solides aux populations insulaires et riveraines en particulier; 
- La sensibilisation autour de mesures d'hygiène et de préservation de la quantité et de la qualité des eaux en fonction des usages.

Dans le cadre de la mise en œuvre de la GIRE et pour l'atteinte des objectifs du millénaire au Burkina Faso, les autorités en charge des ressources en eau prévoient la mise en place de "Comités Locaux de l'Eau : CLE". Pour la promotion d'un développement durable dans la vallée du Sourou, ces "CLE", pourraient s'inspirer de la démarche et des acquis du "Contrat de Rivière Sourou".

\section{Conclusion}

Les résultats obtenus dans cette étude indiquent que les eaux de surface dans la vallée du Sourou sont relativement de bonne qualité au plan physico-chimique et bactériologique pour certains usages essentiellement en période de hautes eaux. Par contre, ces eaux sont en proie à une dégradation croissante, à un risque d'eutrophisation et à une exploitation accrue face à la demande de plus en plus élevée en période de basses eaux. En outre, il apparaît clairement que ces eaux sont impropres à la consommation humaine quelle que soit la période de l'année au regard des normes OMS en vigueur (WHO, 2004). Les sources de pollution des eaux constatées sont essentiellement liées entre autres aux activités domestiques, à l'abreuvement d'animaux et aux activités agricoles au voisinage des cours d'eau et dont les intensités et effets induits sont plus prononcés en saison sèche et en début de saison d'hivernage. Ces dégradations de la qualité des plans d'eau ont sans conteste une influence négative sur la santé des populations et par voie de conséquence sur le développement socioéconomique de la vallée. Les données préliminaires obtenues dans cette étude devraient être plus étoffées par la prise en compte de paramètres plus exhaustifs (métaux lourds toxiques, micropolluants organiques, pesticides,...) et un suivi annuel plus régulier qui permettent de mieux caractériser les eaux de surface dans la vallée Sourou en fonction des usages aux différentes périodes de l'année pour une gestion efficiente et efficace.

\section{REMERCIEMENTS}

Les auteurs remercient la Région Wallonne de Belgique, le Département des Sciences et Gestion de l'Environnement de l'Université de Liège, l'IRSS/CNRST, le LNAE et la COPROD pour leurs appuis financiers et techniques.

\section{REFERENCES}

Bethmont J, Faggi P, Zoungrana TP. 2003. La Vallée du Sourou (Burkina Faso). Genèse d'un Territoire Hydraulique dans l'Afrique Soudano-sahélienne. Harmatan : Paris ; p. 230.

Biney C, Calamari D, Maembé TW, Nyakageni B, Saad MAH. 2005. Bases scientifiques du contrôle de pollution. FAO, Dép. Agric., Bulletin technique, p.16.

Dianou D, Poda JN, Sorgho H, Wango SP, Sondo KB. 2003. Hydraulic planning and schistosomiasis: Case of Sourou in Burkina Faso. Journal of Applied Research in Veterinary Medicine, 1(2): 105-111.

Dianou D, Poda JN, Savadogo LG, Sorgho H, Wango SP, Sondo B. 2004. Parasitoses intestinales dans la zone du complexe hydroagricole du Sourou au Burkina Faso. Vertigo, 5(2): 1-8.

Julien F. 2006. Maîtrise de l'eau et développement durable en Afrique de l'ouest : de la nécessité d'une coopération régionale autour des systèmes hydrologiques transfrontaliers. Vertigo, 7(2).

Kaboré A. 2011. Etude du pouvoir floculant et des qualités épuratoires des graines de Moringa oleifera dans le traitement des 
eaux brutes de consommation en Afrique sub-saharienne : Cas des eaux du Burkina Faso. Mémoire de DEA, Université de Ouagadougou, Ouagadougou, p. 54.

Kouam KGR, Mpakam HG, Ndonwy SA, Bopda SLD, Ekodeck GE. 2006. Gestion intégrée des ressources en eau et objectifs du millénaire pour le développement en Afrique : Cas du Cameroun. Vertigo, 7(2): $1-9$.

Koukounari A, Gabrielli AF, Toure S, Bosque-Oliva E, Zhang Y, Sellin B, Donnelly CA, Fenwick A, Webster JP. 2007. Schistosoma haematobium infection and morbidity before and after large-scale administration of Praziquantel in Burkina Faso. Journal of Infectious Diseases, 196: 659-669.

Leprun JC. 1969. Evolution géomorphologique de la Vallée du Sourou et de ses bordures. Mémoire de maîtrise de géographie, Université de Dakar, Dakar, p. 49.

MAHRH (Ministère de L'agriculture, de l'Hydraulique et des Ressources Halieutiques). 2011. Rapport bilan GIRE Burkina Faso 2010 et perspectives 2011, Ouagadougou, p. 29.

MEA (Ministère de l'Environnement et de l'Eau). 2011. L'impact des engrais et des pesticides sur les ressources en eau au Burkina Faso. Bulletin Technique : Projet GIRE Burkina Faso, Ouagadougou, p. 40.

Nisbet M, Vernaux J. 1970. Composition chimiques des eaux courantes : Discussion et proposition de classes en tant que base d'interprétation des analyses chimiques. Annales de Limnologie, 6(2): 161-190.

Phillips DJH, Daoudy M, McCaffrey SC, Öjendal ARJ. 2006. Trans-boundary Water Co-operation as a Tool for Conflict Prevention and Broader Benefit Sharing, Stockholm, Ministère suédois des Affaires Etrangères, p. 249.
Pléan M, Cissé AS, Kéita K, Doumbia A. 2005. Qualité des eaux du fleuve Niger à Bamako: impacts des activités domestiques. Conférence sur la qualité des eaux de Bamako, Bamako, p. 44.

Poda JN, Sorgho H, Dianou D, Kambou T, Sawadogo B, Sondo B. 2001. Profil parasitologique de la schistosomiase urinaire du complexe hydro-agricole du Sourou au Burkina Faso. Bulletin de la Societé de Pathologie Exotique, 94(1): 2124.

Poda JN, Wango SP, Sorgho H, Dianou D. 2004. Evolution récente des schistosomoses dans le complexe hydroagricole du Sourou au Burkina Faso. Bull. Soc. Pathol. Exot., 97(1): 15-18.

Poda JN. 2007. Les maladies liees a l'eau dans le Bassin de la Volta : Etat des lieux et Perspectives. Volta Basin Focal Project Report No. 4, Ouagadougou, p. 86.

Poda JN, Mwanga J, Dianou D, Garba A, Ouédraogo FC, Zongo D, Sondo KB. 2006. Les parasitoses qui minent les nouveaux pôles de développement au Burkina Faso : cas des schistosomoses et des géohelminthes dans le complexe hydro agricole du Sourou. Vertigo, 17(2): 1-7.

Pouilleute E. 1996. Les phénomènes d'eutrophisation des rivières, des lacs et des réservoirs. Mémoire D.U. en Eau et Environnement, D.E.P., Amiens, p. 40.

Richard S, Rieu T. 2009. Vers une gouvernance locale de l'eau en France: analyse d'une recomposition de l'action publique à partir de l'expérience du schéma d'aménagement et de gestion de l'eau (SAGE) de la rivière Drôme en France. Vertigo, 9(1): 1-46.

Rodier J. 1996. L'Analyse de l'Eau (8 ${ }^{\mathrm{ème}}$ édn). Dunod : Paris.

Rosillon F. 2004. River contract in the Sourou valley (Burkina Faso), Communication at the Academy of African Business and 
Development, April 7-10, Georgia State University, Georgia.

Rosillon F, Vander Borght P, Bado Sama H. 2005. River contract in Wallonia (Belgium) and its application for water management in the Sourou valley (Burkina Faso). Water Sciences and Technology, 52(9): 85-93.

Rosillon F, Bado SH. 2006. Local actions for a global challenge through the experiences of a River contract in Belgium and in Burkina Faso (West Africa). Poster presented at the $4^{\text {th }}$ World Water Forum, Mexico.

Rosillon F. 2007. Le contrat de rivière : un outil de gestion intégrée et participative de l'eau au niveau local à vocation universelle ? Application en Région wallonne de Belgique et au Burkina Faso. Premier colloque international sur la problématique de l'eau en République Démocratique du Congo, Université de Kinshasa, Kinshasa, p. 16.

Some K, Dembele Y, Some L, MillogoRasolodimby J. 2008. Pollution des eaux dans le bassin du Nakambe: cas des réservoirs de Loumbila et de Mogtedo au Burkina Faso. Sud Sciences et Technologies, 16: 14-22.

Tec. 2005. Catalyser le changement : manuel de développement de la gestion intégrée des ressources en eau (GIRE) et des stratégies d'efficience de l'eau. Global Water Partnership. Stockholm, Suède, p. 48.

Traoré I. 2003. Impact des facteurs géographiques sur le développement des Schistosomiases dans la vallée du Sourou.
Mémoire de Maîtrise en Géographie, Université de Ouagadougou, Ouagadougou, p. 119.

Turpin N, Boutems P, Rotillon G. 2003. Restauration de la Qualité des Eaux de Surface : Comparaison des Mécanismes Incitatifs. CEMAGREF-INRA : p. 10.

Un-water/wwap. 2006. L'eau, une responsabilité partagée. Résumé du $2^{\text {ème }}$ Rapport Mondial des Nations Unies sur la mise en valeur des ressources en eau. UNWATER, p. 52.

Wellens J, Traoré F, Niamian MJ, Karambiri H, Diallo M, Compaoré NF, Dakouré D, Derouane J, Tychon B. 2008a. Elaboration du bilan d'eau du bassin versant du Kou et analyse critique des principaux composants. Bulletin Technique: Projet GEeau, BoboDioulasso, Burkina Faso, p. 9.

Wellens J, Diallo M, Gardin N, Midekor A, Compaoré NF, Dakouré D, Karambiri H, Derouane $\mathrm{J}$, Brouyère $\mathrm{S}$, Tychon $\mathrm{B}$. 2008b. Promouvoir des approches innovantes de recherche-développement en matière de GIRE au Burkina Faso. Bulletin Technique : Projet GEeau. BoboDioulasso, Burkina Faso, p. 9.

WHO. 2004. Guidelines for Drinking Water Quality (3rd Edn). WHO. ISBN 92-4154638-7, p. 540. 